\title{
Angle illusion on a picture's surface
}

\author{
SHERIEF HAMMAD, JOHN M. KENNEDY*, IGOR JURICEVIC \\ and SHAZMA RAJANI \\ University of Toronto at Scarborough, 1265 Military Trail, Toronto ON M1C1A4, Canada
}

Received 28 August 2006; accepted 15 March 2007

\begin{abstract}
Shapes on picture surfaces are not seen accurately (Arnheim, 1954). In particular, if they depict 3-D forms, angles between lines on a picture surface are misperceived. To test four theories of the misperception, subjects estimated acute and obtuse internal angles of quadrilaterals. Each quadrilateral was shown alone or as part of a drawing of a cube. The drawings showed the tops of the cubes, tilted at various angles around a horizontal axis. This generated different acute and obtuse angles in the drawings. Compared to a quadrilateral on its own, judgments of the acute and obtuse angles in the cube drawings were biased towards $90^{\circ}$. The bias was present over a wide range of intermediate tilts. The results support a perspective convergence theory and run counter to 'Extreme Foreshortening', Gestalt and Cognitive theories.
\end{abstract}

Keywords: Perspective; illusion; pictures; cubes; angles; Gregory; Arnheim.

\section{INTRODUCTION}

Artists contend with three major sources of misperception. The first makes its presence known when artists judge the visual angles of objects in the scene to be portrayed. Artists resort to holding a pencil or brush at arm's length, aligned with the object. The reason is that the linear size of an object often interferes with impressions of its visual angle.

The second source becomes apparent as the artist draws more and more marks on a picture surface to show more and more of the scene. A mark that looks wrong on its own will often look right as soon as perspective shows the depths in the scene (Arnheim, 1954, p. 97; Kennedy, 1974, p. 37) - and vice versa! This source of error is our concern in the present paper.

To be complete, we should mention a third source. Often pictures that are perfect panoramic projections of the world give remarkably distorted impressions. To the deep concern of wealthy patrons whose goal is pictures that look realistic, since the

\footnotetext{
*To whom correspondence should be addressed. E-mail: kennedy@utsc.utoronto.ca
} 
late Renaissance artists have found that information about the sizes and angles of parts of the scene is often used quite incorrectly by vision, despite the picture being geometrically correct (Juricevic and Kennedy, 2006).

Of these three domains of enquiry about picture misperception, in the present study we single out for attention the picture surface and illusions to do with angles. Elsewhere, perception of individual objects posed at distinctive tilts was discussed (Kennedy, 1974, 1993, 2001). Here, we test theories of an angle illusion on a picture surface by studying the effects that occur across the full range of possible tilts of a square, from close to $0^{\circ}$ to close to $90^{\circ}$.

\section{Perspective and a picture-surface angle illusion}

Apparent distortions have been of interest to theory of art since the late Renaissance and the development of perspective in the 1400s. The crux of constancy research is how we perceive a 3-dimensional form despite it sending many different projections to our eye as we walk around it. How we avoid distortion was crucial to perception philosophy in the 1600s and 1700s in the writings of British Empiricists Locke, Berkeley, Hume, Bacon and Reid, and central to perception psychology since Helmholtz in the 1800s. It was debated at length in Gestalt and ecological theory of pictures in the Twentieth Century (Arnheim, 1954; Gibson, 1979).

Juricevic and Kennedy (2006) analyze distortions of apparent length in panoramic pictures. Kubovy (1986) considers perceived angles at corners of cubes. Gregory (1963) theorized about illusions resulting from unconscious use of perspective. For present purposes, a helpful case in point is two lines forming an $\mathrm{X}$ on the face of a perspective drawing of a cube. The acute and obtuse angles between the two lines depict right angles if the lines are parallel to contours depicting the edges of the face on which they are drawn. At times they may appear to be right angles on the picture surface (Parks, 2001). Indeed, the impetus for the current research stems from a study of EA, a congenitally blind man drawing cubes (Kennedy and Juricevic, 2006). Intriguingly, the Vs representing the corners in EA's drawings of cubes like Fig. 1 look close to $90^{\circ}$ on the page, and equal, though in fact they are $25^{\circ}$ apart, and the acute and obtuse angles differ from $90^{\circ}$ by $10^{\circ}$ to $15^{\circ}$.

To test theories of the misperception of angles in pictures, we presented observers with pictures of cubes at various tilts. Figure 1 may demonstrate the illusion to the reader. Two drawings of cubes in the illustration have quadrilateral tops. Two of the nine quadrilaterals below match the tops but the typical observer is unable to make the correct selections. The top on the left matches row three, column three and the one on the right matches row two, column two. Observers fail even if they describe themselves as judging the acute and obtuse internal angles of the quadrilaterals, the vertical extents of the quadrilaterals or parallel lines of the tops and the lower quadrilaterals. The obtuse topmost internal angle of the cube drawing on the left is $143^{\circ}$. Many observers suggest its closest match in the 9 quadrilaterals is in row one column one - a $130^{\circ}$ angle. Matching its acute angle $\left(41^{\circ}\right)$ with row one, column one's acute angle $\left(54^{\circ}\right)$ is an error of $13^{\circ}$, an error of almost one-third. 

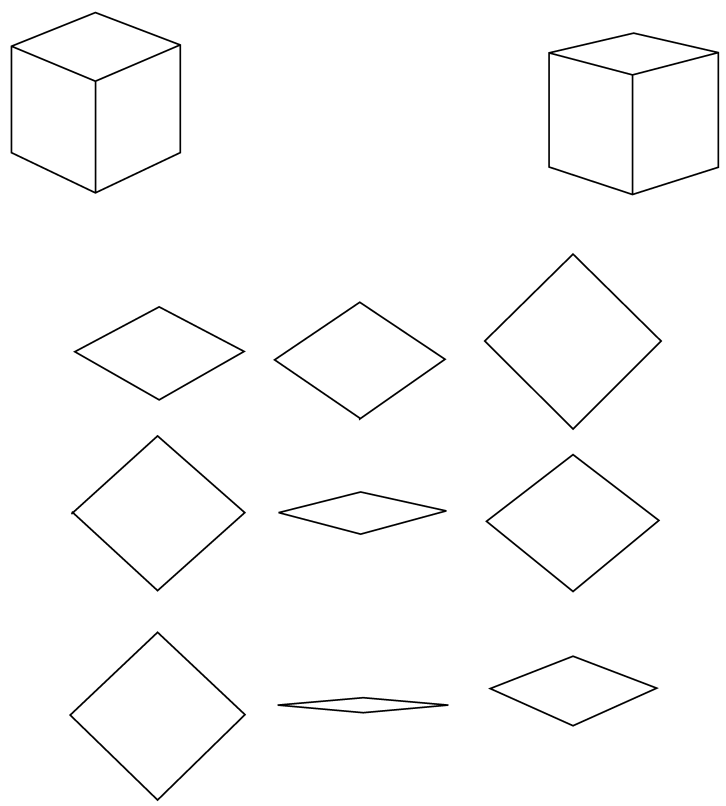

Figure 1. Two of the quadrilaterals correspond to the top faces of the two cubes, but observers misidentify the matches. The top on the left matches row three, column three and the one on the right matches row two, column two.

Projection from a 3-D scene onto a 2-D surface

To introduce four theories about Fig. 1's effects, let us consider projection onto a picture surface.

Perspective projection results in convergence and foreshortening. In particular, in three-point perspective the left and right sides of the drawing of the cube converge, and verticals converge down the page, indicating the cube's tilt and revealing the top of the cube (Fig. 2).

A simple example of convergence downwards would be given in a photograph of the Empire State Building from above the front face of the building with the camera tilted to the vertical. In the photograph the contours depicting the edges of the building would narrow towards the sidewalk.

A tilt in depth produces foreshortening. In the well-known "two tables" illustration (Shepard, 1990), table-tops that are congruent on the picture surface are perceived as unequal because foreshortening changes the width of one and the length of the other. Foreshortening simultaneously depicts depth and creates apparent differences on the picture surface.

Now let us consider what might cause a change in apparent angle on a picture surface. 


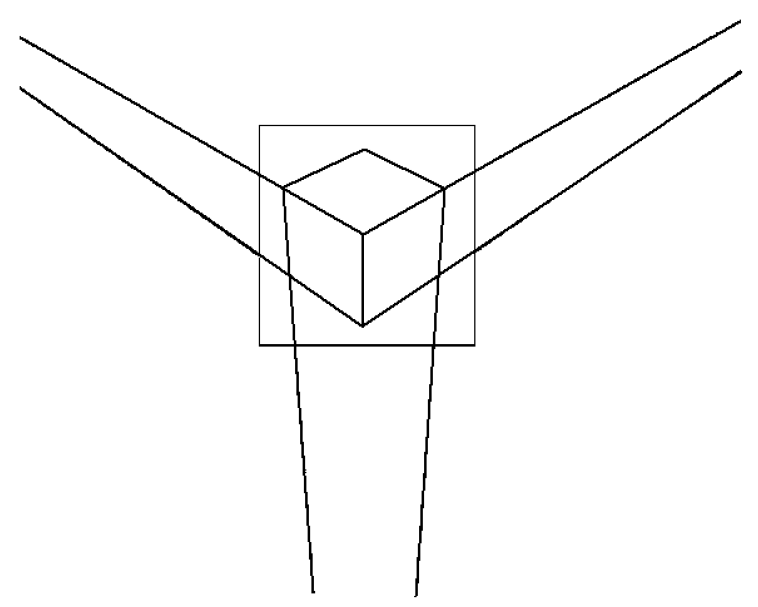

Figure 2. Cube drawn with extended lines to reveal three-point perspective. The left and right sides of the drawing of the cube converge to left and right, respectively. The vertical sides of the cube converge down the page, indicating that the cube is tilted ( $35^{\circ}$ from horizontal).

\section{Convergence, Foreshortening, Cognitive, and Gestalt theories}

Sedgwick (2003) describes pictorial information affecting perception of the surface of a picture, and vice versa, as 'crosstalk' resulting in a compromise between true space and its projection (Arnheim, 1954; Sedgwick and Nicholls, 1993).

The compromise might be due primarily to converging lines on the picture surface. If so, its effect might be most obvious at intermediate tilts, when the converging lines are distinct. Convergence is not obvious when the cube sides are close to parallel to the picture surface, and project lines close to parallel on the picture surface. Likewise, if the cube is highly tilted, the lines for the cube sides are projected as highly foreshortened and the convergence of very short lines is hard to discern. It follows that if convergence of the lines depicting cube sides plays a major role in misperception of Fig. 1, the illusion should be most evident at intermediate angles of tilt. Let us call this Convergence theory.

Alternatively, obvious foreshortening may be the driving force. The further a depicting form is from the depicted form, the more obvious the foreshortening, and the more crosstalk, theory could contend. 'Extreme Foreshortening' would be 'corrected' in this theory. The more extreme the tilt, the larger the crosstalk because extreme tilts involve more foreshortening. If the top of a cube is pictured as close to parallel to the picture surface it projects a form close to a square, with minimum foreshortening.

Alternatively, an angle illusion could be a top-down, Gestalt effect. When physical conditions approach a 'simpler' shape, a percept with symmetry, equality, parallels, and orthogonality is favoured, Gestaltists argue. Visual processes respond to higher-order patterns in stimuli, albeit patterns quite incidental to perspective. "Perception transcends constantly and routinely the mere mechanical recording of sensory raw material" (Arnheim, 1980). "Projective distortions are observed only 
by the select few who have been trained to watch out for them" (Arnheim, 1986). In Gestalt theory, figures that are close to a 'good form' in shape are perceived in ways related to that shape. Four $90^{\circ}$ angles in a quadrilateral would make an especially good form. If so, angles in the input approaching $90^{\circ}$ may be seen as $90^{\circ}$.

In Cognitive theory, top-down effects arise from psychological representations of familiar angles. An interpretation of a 2-dimensional drawing as a cube showing $90^{\circ}$ corners could feed down to the perceptual input and make the acute and obtuse Vs be seen as $90^{\circ}$. If the $\mathrm{V}$ means $90^{\circ}$, it is seen as $90^{\circ}$, in a strong version of this theory. Peterson and Gibson (1993) argue perceptual input and cognitive information are processed in parallel. If recognition influences perception, angles close to $90^{\circ}$ on the page might indeed be seen as $90^{\circ}$. However, it may be that angles on the page very much larger than $90^{\circ}$ would be unaffected. They might readily be recognized to be far from $90^{\circ}$.

In sum, Convergence theory predicts illusions at intermediate tilts, 'obvious' foreshortening theory focuses on tilts producing 'Extreme Foreshortening', and the versions of Gestalt theory and Cognitive theory considered here both predict strong illusions as angles on the page approach $90^{\circ}$.

To test these four theories, we presented observers with quadrilaterals either alone, or as the top faces of cubes. They estimated the angles between the lines on the flat surface of the screen. By drawing cubes tilted around a horizontal axis, we changed the internal angles in the top face quadrilaterals (and congruent quadrilaterals presented alone).

\section{Method}

Participants. Twelve participants (8 male, mean age 20, range 2 years) were recruited from a first year psychology class at the University of Toronto at Scarborough. Each had normal or corrected-to-normal vision and was naïve to the purpose of the experiment.

Stimuli. All stimuli were generated in a bespoke program called Angle Grinder, written in Java programming language and using 'Java 3D API' to render the cubes. They were presented to all participants on a 15 in. $(38.1 \mathrm{~cm})$ computer monitor. Cubes were drawn in linear perspective with white lines $1 \mathrm{~mm}$ thick, on a black background. Three faces of each cube were shown; the top and two sides. The convex corner between the two side faces was toward the observer, and the $\mathrm{Y}$ intersection between the sides and the top was located in the centre of the screen; hence the top face was depicted as being tilted towards the observer. The cubes were drawn at nine different orientations. The top face was tilted vertically from the horizontal in $10^{\circ}$ increments between $5^{\circ}$ and $85^{\circ}$. At a tilt of $5^{\circ}$ the top was highly foreshortened. It resembled a slim, highly pointed diamond, long axis leftright on the screen. The left-most $\mathrm{V}$ shape was highly acute, $9^{\circ}$. Correspondingly, the top-most $\mathrm{V}$ shape on the screen was highly obtuse, $171^{\circ}$. At a tilt of $85^{\circ}$ the projection of the top was very close to a square. Each tilt was a rotation out of the 

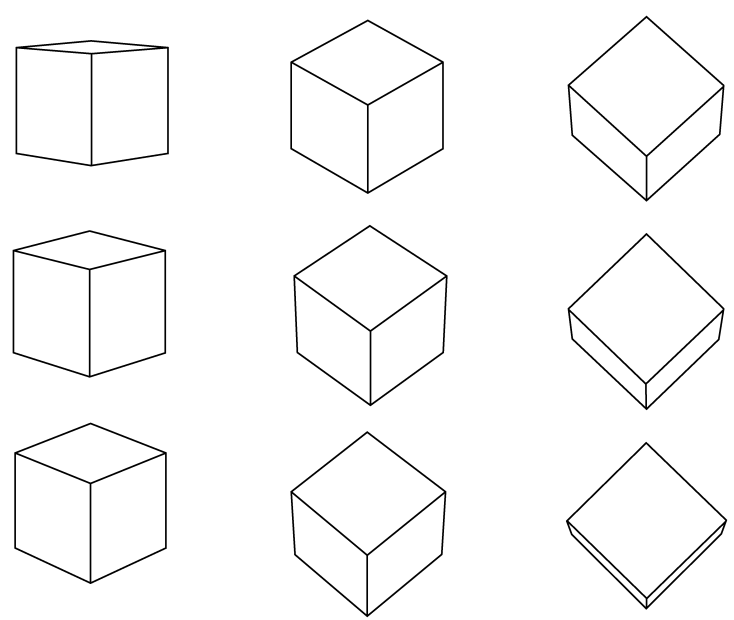

Figure 3. Orientation of cube stimuli through nine tilt angles from the horizontal.

picture plane about the $\mathrm{Y}$ intersection between the three visible faces (Fig. 3). As a result of this manipulation the upper-most angle of the quadrilateral shown at the top of the cube varied from $171^{\circ}$ to $91^{\circ}$, and the left-most angle of the quadrilateral varied from $9^{\circ}$ to $89^{\circ}$. Because the cubes were depicted in linear perspective, each side diminished in size as it receded away from the viewer. The resulting top-most quadrilateral does not constitute a perfect parallelogram, and hence adjacent corners do not always add up to $180^{\circ}$.

Participants were shown each of the 9 cube drawings five times in a randomised order. Accordingly, no two participants received the same order of tilt rotations and associated picture angles. The stimuli were presented in two blocks. In one, the quadrilateral whose angles were to be judged was shown as the top quadrilateral of a cube (cube condition). In the other, the quadrilateral was shown entirely on its own, that is, with the lines for the cube sides removed (quadrilateral condition). Half the participants received the cube condition first, and the other half were shown the quadrilateral condition first.

The quadrilaterals presented alone were the same as the ones depicting the cubes' top faces, with the same angle combinations (Fig. 4). In the trials, the order of the quadrilaterals was the same as the order of the cube tops that they copied.

Procedure. Participants were given instructions orally. For practice, they were first asked to estimate the angles between lines on a piece of paper (range $120^{\circ}$ ). Five practice drawings were presented. They were made of white lines approximately $1 \mathrm{~mm}$ thick, on a black background, in order to be similar to the experimental stimuli. All participants were accurate to within $10^{\circ}$ mean error on all judgments.

Participants' heads were $77 \mathrm{~cm}$ from the computer screen, using a chin rest to help ensure that the eye-level of each person was approximately at the centre of the 


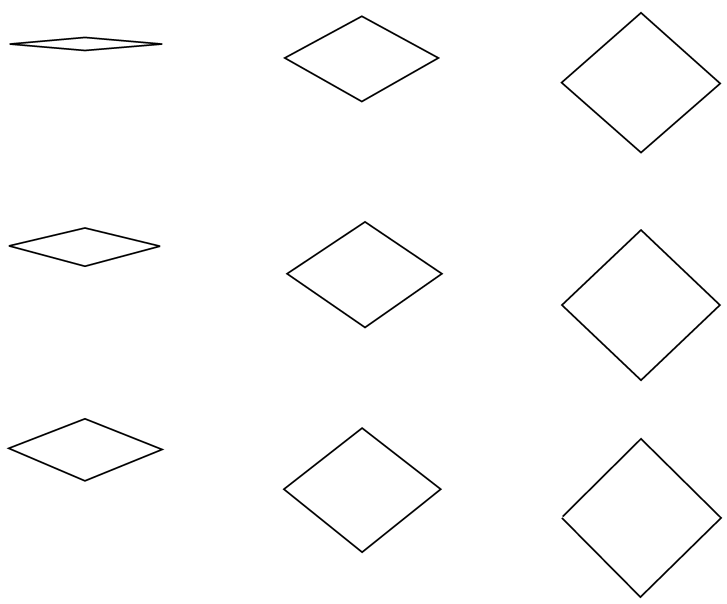

Figure 4. Orientation of quadrilateral stimuli through nine tilt angles from the horizontal.

screen. The participants were informed that they would see pictures of quadrilaterals on the computer screen, with some being taller and some being shorter. They would be required to estimate the top-most or left-most angle (hereafter referred to as the 'obtuse' and 'acute' angles respectively) in each picture on the flat surface of the screen. Half the participants were asked for the obtuse angle in the first 45 trials, and half were asked for the acute angle first. Each stimulus was shown for $5 \mathrm{~s}$, after which participants were prompted by a message on the screen to enter their estimations of these angles on the keypad. During instruction, participants were shown sample pictures (taken from the actual stimuli) of one quadrilateral and one cube presentation. To ensure the subjects attended to the correct angle in the presentations, the requisite angles were indicated with an arc. After five presentations of each quadrilateral, and a total of 45 trials, the program instructed the participant to call the experimenter, and instructions were given for the second block.

In the cube condition participants were informed that they would see pictures made up of three quadrilaterals, and that these would be of different dimensions. They were asked to estimate the obtuse or acute angle in each picture depending on their group assignment. They were shown sample pictures taken from the actual stimuli and shown which angles they were to estimate. For half of the participants, the cube and quadrilateral conditions were reversed in order (cubes first, then quadrilaterals).

At no time did the experimenter make mention of a 'cube' to describe the drawings. This was to discourage participants from judging the 3-D object depicted in the display, and reporting only right-angles in the stimuli. In addition, participants were asked to estimate the size of angles on the left of the screen, and not the angles on the right side of the screen, as this might have coerced some subjects to report right-angles. 


\section{Results}

Judgments of the angles in cube drawings were heavily biased towards $90^{\circ}$ (Fig. 5), with the obtuse angles' mean error $15^{\circ}$ less than physically correct, and the acute angles $9^{\circ}$ greater than veridical (Table 1 ). The maximum mean error was $31.2^{\circ}$, for judgments of the $157^{\circ}$ angle, reported as a mean of $125.8^{\circ}$. Errors in judging quadrilaterals were much smaller (mean error $0.61^{\circ}$ greater than veridical for acute angles and $5^{\circ}$ less than accurate for obtuse angles). Errors in the responses were analysed for acute and obtuse angles separately.

For all statistical tests here, the criterion was $p<0.05$.

Acute angles. In a 2 (cube vs quadrilateral) $\times 9\left(9^{\circ}, 26^{\circ}, 41^{\circ}, 54^{\circ}, 65^{\circ}, 75^{\circ}, 80^{\circ}\right.$, $87^{\circ}, 89^{\circ}$ ) within-subjects ANOVA, acute angles invoked greater errors in drawings of cubes than in quadrilaterals alone, $F(1,8)=8.1, M S E=367.8$.

In paired-sample $t$-tests, judgments of acute angles in cubes were different from those in quadrilaterals at $26^{\circ}(t(8)=2.16), 41^{\circ}(t(8)=2.81), 54^{\circ}(t(8)=2.68)$, $65^{\circ}(t(8)=2.52), 75^{\circ}(t(8)=3.37), 80^{\circ}(t(8)=2.26), 87^{\circ}(t(8)=2.37)$.

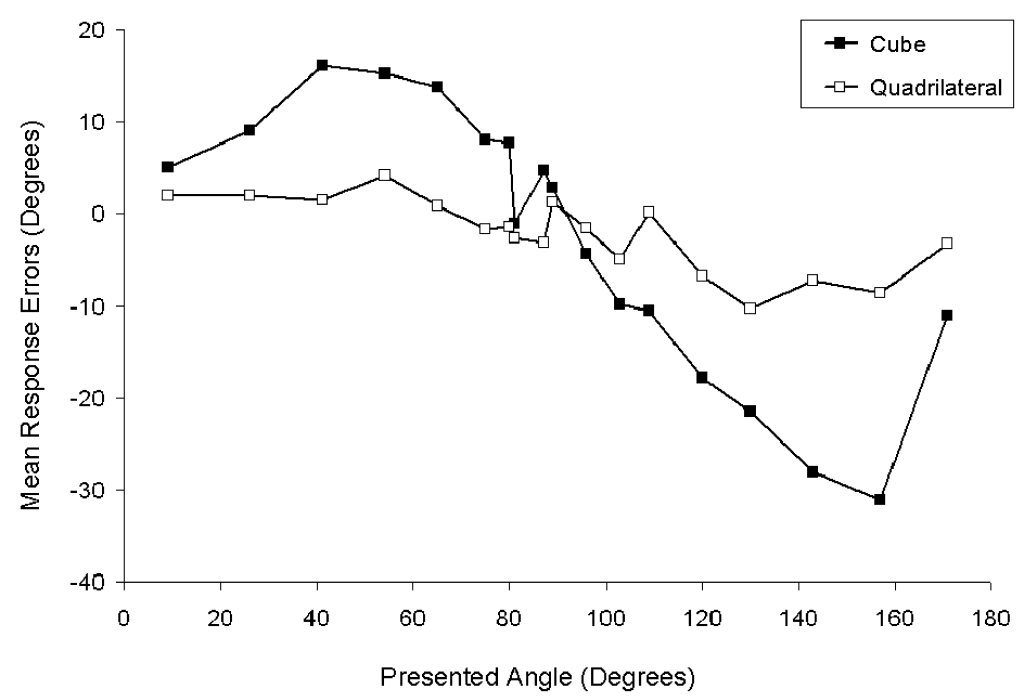

Figure 5. Mean errors in response to acute and obtuse angles in cube drawings and in quadrilaterals on their own.

Table 1.

Mean response errors in angle estimations

\begin{tabular}{lllrr}
\hline & Cube & $\begin{array}{l}\text { Quadrilateral } \\
\text { Obtuse }\end{array}$ & $\begin{array}{l}\text { Cube } \\
\text { Acute }\end{array}$ & \multicolumn{2}{c}{$\begin{array}{l}\text { Quadrilateral } \\
\text { Acute }\end{array}$} \\
\hline Mean reported angle $^{\circ}$ & 109.40 & 119.44 & 67.61 & 59.05 \\
Mean error $^{\circ}$ & -15.04 & -5.00 & 9.16 & 0.61 \\
\hline
\end{tabular}


Obtuse angles. In a 2 (cube $v s$ quadrilateral) $\times 9$ (presented angle: $91^{\circ}, 96^{\circ}$, $103^{\circ}, 109^{\circ}, 120^{\circ}, 130^{\circ}, 143^{\circ}, 157^{\circ}, 171^{\circ}$ ) within-subjects ANOVA, judgments of obtuse angles in cubes were smaller than judgments of angles in quadrilaterals, $F(1,8)=14.7, M S E=278.1$.

Paired-sample $t$-tests found significant differences between cube and quadrilateral conditions for $109^{\circ}(t(8)=-2.79), 120^{\circ}(t(8)=-3.00), 130^{\circ}(t(8)=2.37), 143^{\circ}$ $(t(8)=4.54), 157^{\circ}(t(8)=3.79) 171^{\circ}(t(8)=2.48)$.

\section{DISCUSSION}

Errors are greater for the top face in a drawing of a cube than for a congruent quadrilateral. For the top face, obtuse angles are underestimated and acute angles overestimated, closer to $90^{\circ}$ than is the case, over a wide range of angles (26 to $87^{\circ}$ and $109^{\circ}$ to $171^{\circ}$ ). This supports the Convergence hypothesis. The maximum error for obtuse angles is at $157^{\circ}$, and for acute angles is $41^{\circ}$, fully $116^{\circ}$ apart, from a possible range of $162^{\circ}$ tested. Indeed at $9^{\circ}$ and $171^{\circ}$ the errors were still $5^{\circ}$ and $11^{\circ}$, respectively.

The Extreme-Foreshortening, Gestalt and Cognitive hypotheses are not supported. Errors are not concentrated at the extremes (neither the $5^{\circ}$ nor the $85^{\circ}$ tilts). Of interest, the judgments are attracted only to $90^{\circ}$, not to $0^{\circ}$ or $180^{\circ}, 45^{\circ}$ and $135^{\circ}$, other possible components of good form. If these were attractors, angles on either side of $45^{\circ}$ and $135^{\circ}$ would be judged as $45^{\circ}$ and $135^{\circ}$. At $41^{\circ}$ the error is large $\left(16^{\circ}\right)$ and takes the judgment $12^{\circ}$ above $45^{\circ}$. Also at $54^{\circ}$ the judgment is $69^{\circ}$, which is towards $90^{\circ}$ and not towards $45^{\circ}$. Likewise, at $130^{\circ}$, the judgment is $110^{\circ}$, away from $135^{\circ}$. At $143^{\circ}$, the error is large $\left(28^{\circ}\right)$ and takes the response below $135^{\circ}$. Similarly, the error at $157^{\circ}$ is large $\left(31^{\circ}\right)$ and makes the judgment lower than $135^{\circ}$. In sum, the errors are attracted solely to $90^{\circ}$ and distributed across a wide range.

\section{Acute angles}

To ensure the sides of the cube were visible, the largest acute angle in the top quadrilateral was $89^{\circ}$. With a decrease in the presented angle from $89^{\circ}$ to $75^{\circ}$ judgments of the quadrilateral change minimally, but there are increasingly large errors in response to the cube drawings. Indeed, at $75^{\circ}$, the errors are $8^{\circ}$ towards $90^{\circ}$ for the cube drawing but $2^{\circ}$ away for the quadrilateral. The most erroneous overestimation $\left(16^{\circ}\right)$ of the acute angle in the cube drawing is made at $41^{\circ}$ (and the quadrilateral at $41^{\circ}$ yields an error of only $2^{\circ}$ ). At $26^{\circ}$, the error for the cube drawing is still $9^{\circ}$. At a presentation angle of $9^{\circ}$ the error in the cube drawing is more than half $-5^{\circ}$. From $41^{\circ}$ to $9^{\circ}$, errors are likely decreasing smoothly to zero at an acute angle limit of $0^{\circ}$ where the cube drawing and the quadrilateral would be identical. 


\section{Obtuse angles}

To ensure side faces of the cube were evident, the largest tilt presented was $85^{\circ}$ from the horizontal. This resulted in an obtuse angle of $91^{\circ}$ being projected on to the screen. At this angle, there is no significant difference in angle judgment between cube and quadrilateral conditions and errors are small.

As the angle presented on the screen increased to $109^{\circ}$ the error in judging the screen angle increased to $-10^{\circ}$ for angles in cube drawings, but was slightly positive for response to quadrilaterals, a significant difference. The error did not take the judgment all the way to $90^{\circ}$, failing to support Gestalt and Cognitive predictions. At $120^{\circ}$ and beyond, judgments of quadrilaterals are still fractions of the errors induced by cube drawings. Beyond $120^{\circ}$, judgments of quadrilaterals are smaller than the presented angles. It may be the quadrilateral results simply reflect a response bias towards lower numbers than $130^{\circ}, 143^{\circ}, 157^{\circ}$ and $171^{\circ}$ and it may be that subjects are more accurate in judging acute angles, but for present purposes the point to note is the large discrepancy between the judgments of the quadrilateral and the cube drawing.

Errors at $171^{\circ}$ are less than those at $157^{\circ}$, though still notable $\left(11^{\circ}\right)$. Likely the errors for obtuse angles approach zero smoothly as a limit at $180^{\circ}$ at which the cube drawing would be identical to the quadrilateral.

\section{Theories}

Obvious 'Extreme-Foreshortening', Gestalt and Cognitive theories argue a form with $90^{\circ}$ corners is imposed on lower-order perceptual processes. What is imposed is a represented form, a simple organization, or a recognized object. However, the errors do not take the form of stating $90^{\circ}$ degrees itself. Large errors occur at intermediate tilts, and the responses are merely towards $90^{\circ}$. Rather than imposition of a standard form, the results suggest a compromise.

The Convergence theory focuses on a variable on the picture surface that becomes more evident as tilt increases, and then less evident as tilt increases further. This characterizes the growth and decline of error with tilt more aptly than the other theories.

Since it does not introduce ideas about representation, the Gestalt theory has a problem not faced by the others. It has trouble explaining the remarkable difference between pictures of squares and actual quadrilaterals. It has no reason for distinguishing the two.

Though the results favour the Convergence theory described here, caveats need to be mentioned. Firstly, the theory cannot predict the amount of error that converging lines may induce at various lengths or rates of convergence. As our understanding of vision's use of perspective develops (Juricevic and Kennedy, 2006), precise prediction may become possible. There should be an optimum rate of convergence (since at one extreme, lines are parallel, and at the other they form a straight line), 
and an optimum length (since the convergence of very short lines is hard to see, and to be very long the lines have to be parallel).

A second caveat is that foreshortening theories are more flexible than the obvious 'extreme-foreshortening' one considered here. In parallel projection, faces of objects are foreshortened but not converged. Entirely in the absence of convergence, at intermediate tilts foreshortening may be sufficient to create misperception of the angles in the depicting forms.

A third caveat is that foreshortening and convergence work together and are confounded in natural optic perspective, and misperception of picture surface elements may be due to their collaboration in spatial information. If so, the issue may not be either foreshortening or convergence per se. Alternatively, foreshortening and convergence might work independently. Indeed, they might summate in producing misperception.

A fourth caveat is that projection rays and object tilt are independent factors. For example, parallel projection is typically taken to be orthogonal to the picture surface. But it can be oblique. The tilt of the depicted surface to the screen can be fixed while the orientation of the projection rays is varied. Different kinds of projections would repay study. They could distinguish the role each factor plays.

A fifth caveat is that we have considered particular versions of Gestalt and Cognitive theories. Alternative Gestalt and Cognitive versions might not be difficult to invent.

In conclusion, tilts intermediate between $5^{\circ}$ and $85^{\circ}$ produced angle illusions on a picture surface. Though Renaissance linear perspective geometry carries realistic depth information, its features affect perception of the elements on the picture surface. While there are still several factors to consider, a hypothesis about perspective convergence was supported against three rivals.

\section{Acknowledgements}

We thank high-school science teachers Seth Bernstein and Joe Hockin (members of TSTOP, Ontario, 2006) for comments on theories of the angle illusion.

\section{REFERENCES}

Arnheim, R. (1954). Art and Visual Perception. University of California Press, Berkeley, USA.

Arnheim, R. (1980). A plea for visual thinking, Critical Inquiry 6, 489-497.

Arnheim, R. (1986). New Essays on the Psychology of Art. University of California Press, Berkeley, USA.

Gibson, J. J. (1979). The Ecological Approach to Visual Perception. Houghton-Mifflin, Boston, USA. Gregory, R. L. (1963). Distortion of visual space as inappropriate constancy scaling, Nature 199, 678-680.

Juricevic, I. and Kennedy, J. M. (2006). Looking at perspective pictures from too far, too close, and just right, J. Exper. Psychol.: General 135, 448-461.

Kennedy, J. M. (1974). A Psychology of Picture Perception. Jossey-Bass, San Francisco, USA.

Kennedy, J. M. (1993). Pictures to Touch. Yale Press, New Haven, USA. 
Kennedy, J. M. (2001). Smart geometry!, in: Looking at Looking, Parks, T. (Ed.), pp. 31-50. Sage, London, UK.

Kennedy, J. M and Juricevic, I. (2005). Object constancy: object orientation affects relative depth in perspective pictures, in: Studies in Perception and Action VIII, Heft, H. and Marsh, K. L. (Eds), pp. 249-253. Erlbaum, Mahwah, USA.

Kennedy, J. M. and Juricevic, I. (2006). Foreshortening, convergence and drawings from a blind adult, Perception 35, 847-851.

Kubovy, M. (1986). The Psychology of Perspective and Renaissance Art. Cambridge University Press, Cambridge, UK.

Parks, T. (2001). Looking at Looking. Sage Press, London, UK.

Peterson, M. A. and Gibson, B. S. (1993). Shape recognition contributions to figure-ground organization in three-dimensional displays, Cognit. Psychol. 25, 383-429.

Sedgwick, H. A. (2003). Relating direct and indirect perception of spatial layout, in: Looking Into Pictures: An Interdisciplinary Approach to Pictorial Space, Hecht, H., Schwartz, R. and Atherton, M. (Eds), pp. 239-299. MIT Press, Cambridge, USA.

Sedgwick, H. A. and Nicholls, A. L. (1993). Cross-talk between the picture surface and the pictured scene: effects on perceived shape, Perception 22 (supplement), 109.

Shepard, R. N. (1990). Mind Sights: Original Visual Illusions, Ambiguities, and Other Anomalies, With a Commentary on the Play of Mind in Perception and Art. Freeman, New York, USA. 